\title{
Ellipsometric studies of microscopic surface roughness of CdS thin films
}

\author{
SUNNY MATHEW and K P VIJAYAKUMAR \\ Department of Physics, Cochin University of Science and Technology, Cochin 682 022, India \\ MS received 2 November 1993; revised 14 June 1994
}

\begin{abstract}
Analysis of changes in surface roughness of CdS thin films with preparation temperature was carried out using variable angle spectroscopic ellipsometry (VASE). The films studied were prepared by spray pyrolysis technique, in the substrate temperature range $200-360^{\circ} \mathrm{C}$. The VASE measurements were carried out in the visible region below the band gap $\left(E_{\mathrm{g}}=2.4 \mathrm{eV}\right)$ of $\mathrm{CdS}$ so as to reduce absorption by the film. The thickness of the films was in the range $500-600 \mathrm{~nm}$. Bruggeman's effective medium theory was used for analysis of the surface roughness of the film. The roughness of the film had a high value $(\sim 65 \mathrm{~nm})$ for films prepared at low temperature $\left(200^{\circ} \mathrm{C}\right)$ and decreased with increase in substrate temperature. This reached minimum value $(\sim 27 \mathrm{~nm})$ in the temperature range $280-300^{\circ} \mathrm{C}$. Thereafter roughness increased slowly with temperature. The growth rate of the films was calculated for different temperature ranges. It was found that the deposition rate decreases with the increase in substrate temperature and have an optimum value at $300^{\circ} \mathrm{C}$. Above this temperature deposition rate decreased sharply. The scanning electron micrograph (SEM) of the film also showed that the film prepared at $280-300^{\circ} \mathrm{C}$ had very smooth surface texture.
\end{abstract}

Keywords. Ellipsometry; surface roughness; CdS thin films; spray pyrolysis.

\section{Introduction}

At present CdS thin films are widely used as the window material in several CdS

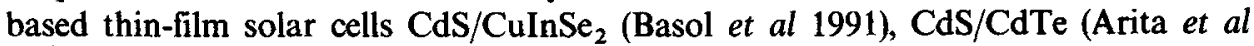
1991). Basol et al (1991) reported that the chemically prepared CdS film is a more ideal window material in thin-film solar cells. They have used ellipsometry for the calculation of optical constants of CdS films. The main objective of the study of polycrystalline CdS thin-films deposited by spray pyrolysis technique has been preparation of film with smooth surface, high transparency and high electrical conductivity in order to increase the efficiency of solar cells. CdS films are prepared by using different techniques such as vacuum evaporation (Kakayama et al 1976), MBE (Shay et al 1975), spray pyrolysis (Cameron et al 1979), CBD (Kaur et al 1981), etc. The optical properties of $\mathrm{CdS}$ thin films are determined to a large extent by the microstructure of the film (Chopra and Das 1983). Detailed studies were reported by various groups on electrical and structural properties of CdS film prepared by spray pyrolysis technique (Banerjee et al 1978; Gupta and Agnihotri 1978; Kwok 1980; Albin and Risbud 1987; Valyommana et al 1992), but fewer reports are available in the case of optical and surface properties of these films prepared by spray technique (Chow et al 1981; Escosura et al 1984; Nolly et al 1987).

In this work we have calculated the changes in surface roughness of CdS film prepared at different temperatures in the range $200-360^{\circ} \mathrm{C}$. The variation in surface roughness was analysed using ellipsometry which is a highly sensitive and nondestructive optical technique for determining the optical and structural properties of thin films (Azzam and Bashara 1977). It is very sensitive to surface irregularities like 
surface roughness, interdiffusion and interlayer formation in multilayer thin-film systems (Azzam and Bashara 1977). The CdS film analysis in the present work was carried out using variable angle spectroscopic ellipsometry (VASE) and we have calculated the changes in surface roughness as well as deposition rate with preparation temperature.

\section{Theory}

Ellipsometry is an optical characterization technique. In this the change in polarization state of light that occurs upon non-normal reflection from the surface of the sample is measured and interpreted to determine the physical properties of the sample. The sample properties can be obtained from the optical model which relates the ellipsometric parameter $\rho$ to the physical properties of the sample through a set of mathematical relations. For example, for a multilayer system (Azzam and Bashara 1977),

$$
\rho=\rho\left\{\varepsilon_{\mathrm{a}}, \ldots, \varepsilon_{j}, \ldots, \varepsilon_{\mathrm{s}}, \ldots, d_{j}, \ldots, \phi, \lambda\right\},
$$

where $\varepsilon_{\mathrm{a}}$ and $\varepsilon_{\mathrm{s}}$ are the dielectric constants of air and substrate and $\varepsilon_{j}$ that of the $j$ th layer, $d_{j}$ the thickness of the $j$ th layer, $\phi$ the angle of incidence, and $\lambda$ the wavelength. The parameters are determined by least-square regression analysis. Theoretical values of the ellipsometric parameters $\psi_{\mathrm{c}}$ and $\Delta_{\mathrm{c}}$ are calculated using Fresnel's reflection coefficients of the optical system. The experimental $\psi_{\mathrm{e}}$ and $\Delta_{\mathrm{e}}$ are calculated from the intensity of light reflected from the thin-film system for different azimuths of analyser. The best-fit model is calculated from the unbiased estimator values obtained from the ellipsometric calculation (Vedam et al 1985). A detailed theory of ellipsometry is given elsewhere (Vijayakumar 1991a).

If the layer is not perfectly filled with a single material but has voids or inclusions of a different material, the optical properties of the mixed layer are no longer determined by the optical constants of one of the constituent materials alone. In the present study the top surface layer of the sample can be considered as a mixture of two components, i.e. air and CdS thin film. If $\varepsilon_{\mathrm{a}}$ and $\varepsilon_{\mathrm{b}}$ are the dielectric constants of the two components of the mixture layer and $\varepsilon_{\mathrm{c}}$ that of the mixed layer, the Bruggeman's effective medium approximation is given as (Bruggeman 1935)

$$
f \frac{\varepsilon_{\mathrm{a}}-\varepsilon_{\mathrm{c}}}{\varepsilon_{\mathrm{a}}-2 \varepsilon_{\mathrm{c}}}+(1-f) \frac{\varepsilon_{\mathrm{b}}-\varepsilon_{\mathrm{c}}}{\varepsilon_{\mathrm{b}}-2 \varepsilon_{\mathrm{c}}}=0,
$$

where $f$ is the volume fraction of the component a.

\section{Experimental}

The CdS thin films were prepared by spraying aqueous solution of $\mathrm{CdCl}_{2}$ and thiourea $(0.01 \mathrm{M})$ on glass substrates kept at different preparation temperatures. Atomization of the chemical solution into a spray of fine droplets is effected by a spray nozzle, with compressed air as carrier gas. The spray rate was kept at $12 \mathrm{~cm}^{3} / \mathrm{min}$. The details of the sample preparation are given elsewhere (Vijayakumar 1991b). The substrate was a clean glass slide without any additional polishing. The back side of each sample was roughened to eliminate the backside reflection. All measurements were done at 
room temperature keeping the sample exposed to the ambient. The ellipsometer was of photometric type with polarizer-system-analyser (PSA) arrangement (Azzam and Bashara 1977). A detailed description of the ellipsometric system and calculation is given elsewhere (Vijayakumar 1991a). The analysis of double-layer thin-film system is done using a FORTRAN program incorporating Bruggeman's effective medium theory. Multiple-angle measurements were performed at all wavelengths with angle of incidence range $60-75^{\circ}$ at an angle interval of $2.5^{\circ}$, which is found to be the most sensitive region of the present CdS thin-film system (Sunny and Vijayakumar 1984).

\section{Results and discussion}

The surface of CdS thin film prepared by spray pyrolysis technique is not smooth and has a microscopically rough top surface (Sunny and Vijayakumar 1994). In order to analyse the relation of this roughness with the substrate temperature at which the film is prepared, the film is assumed to have different layers as depicted in figure 1. Here the top layer contains the rough portion of the film as well as air and hence it has an effective refractive index as described in $\$ 2$. The thickness of the top layer is taken to be $d_{1}$, which is equal to the rms value of the height of the surface irregularities of the film. Hence the measure of this thickness gives direct information about the average height of roughness of the film.

Now using VASE the variation of $d_{1}$ for film prepared at different substrate temperatures is measured and shown in figure 2 . From this figure it can be understood that the film prepared at low temperature has high surface roughness. This value decreases with increase in substrate temperature and reaches a minimum at $\sim 280-300^{\circ} \mathrm{C}$ and thereafter the roughness value increases slowly with temperature. Figure 4 shows SEM photographs of surface roughness of CdS films prepared at different temperatures. From the SEM photographs it is clear that the CdS film prepared at $300^{\circ} \mathrm{C}$ has very smooth surface and below and above this temperature the roughness value increases as indicated by VASE measurements in figure 2 . The variation in the surface roughness can be explained from the growth mechanism of the spray-coated CdS thin films.

Figure 3 shows the deposition rate $r(\AA / \mathrm{min})$ of $\mathrm{CdS}$ film at different substrate temperatures in the range $200-360^{\circ} \mathrm{C}$. It can be seen that the deposition rate decreases with increase in substrate temperature. But the decrease is slow when the substrate temperature is in the range $200-300^{\circ} \mathrm{C}$. The deposition rate decreases at a much

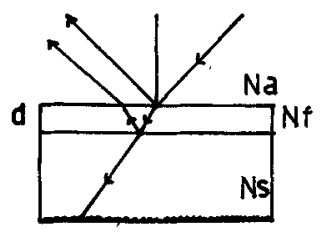

(a)

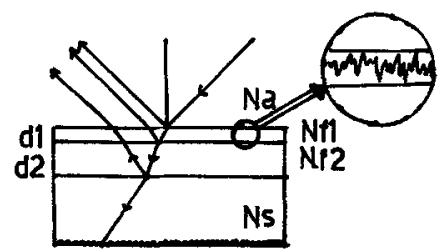

(b)

Figure 1. Model of the film used for the VASE analysis. This film is assumed to have two layers in which the top one contains the surface irregularities. (a) Air/film/substrate system, (b) air/rough layer/film/substrate system. $d, d_{1}$ and $d_{2}$ are the thickness of different layers. $N_{\mathrm{a}}, N_{f}, N_{f 1}, N_{f 2}$ and $N_{\mathrm{s}}$ are the complex refractive indices of different layers. 


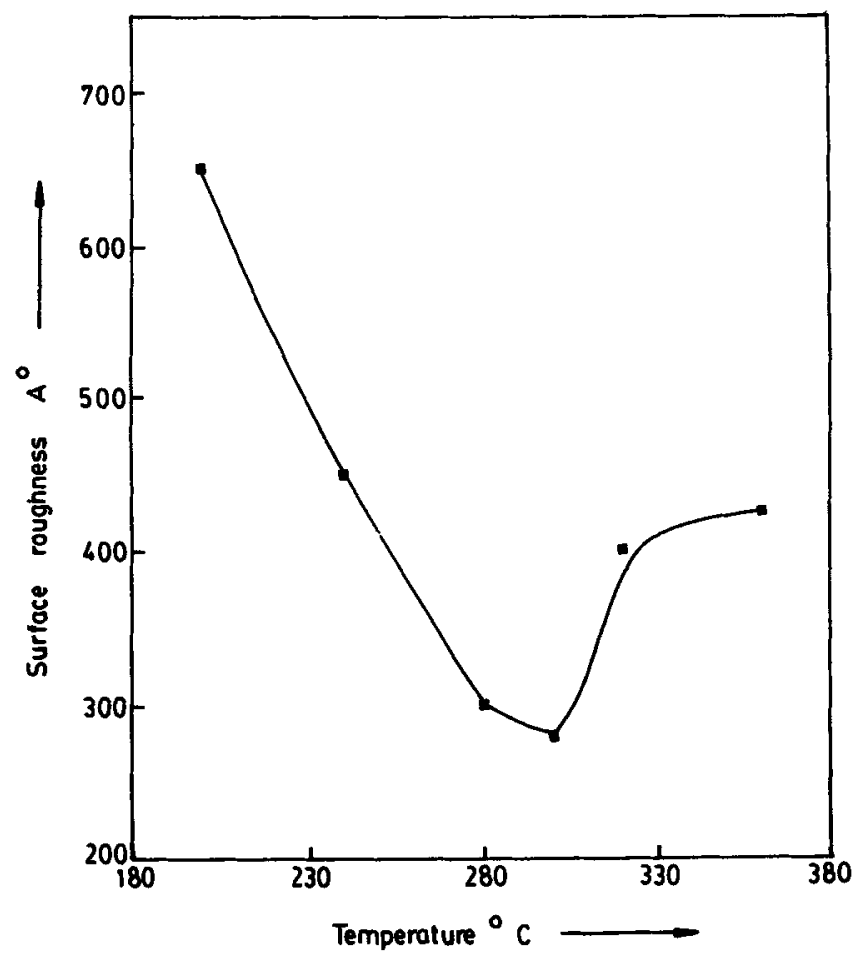

Figure 2. Variation of thickness of rough surface layer (i.e. average height of surface roughness) with temperature.

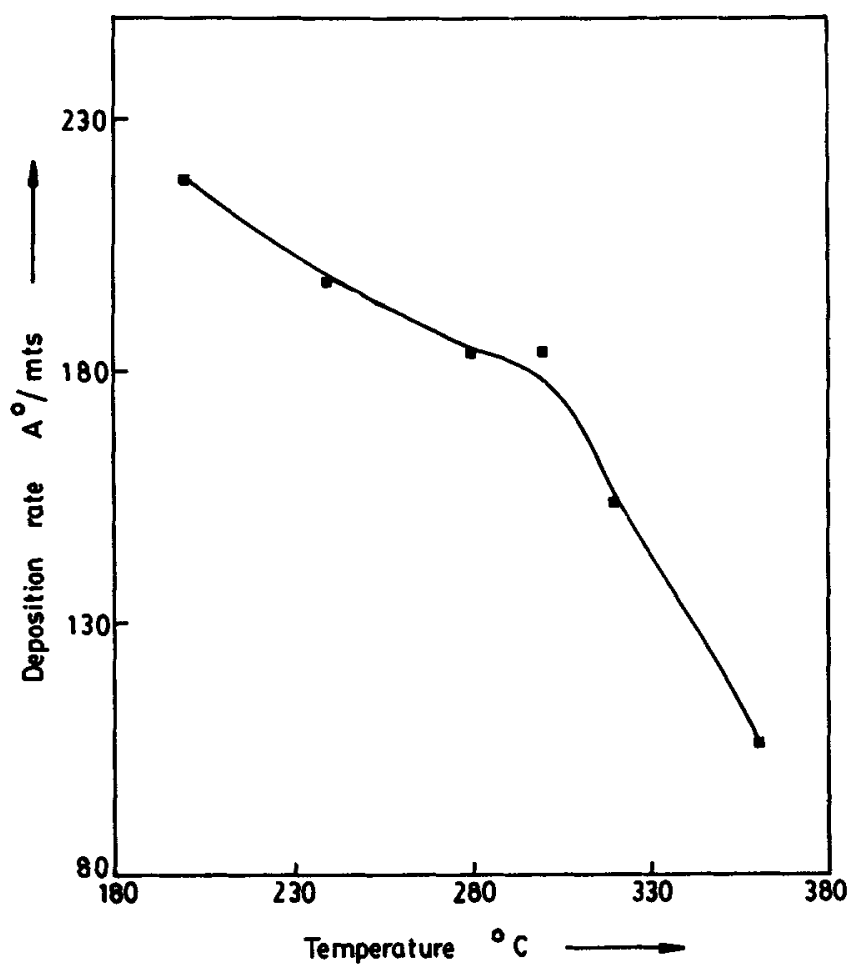

Figure 3. Variation of deposition rate of CdS film with substrate temperature. 

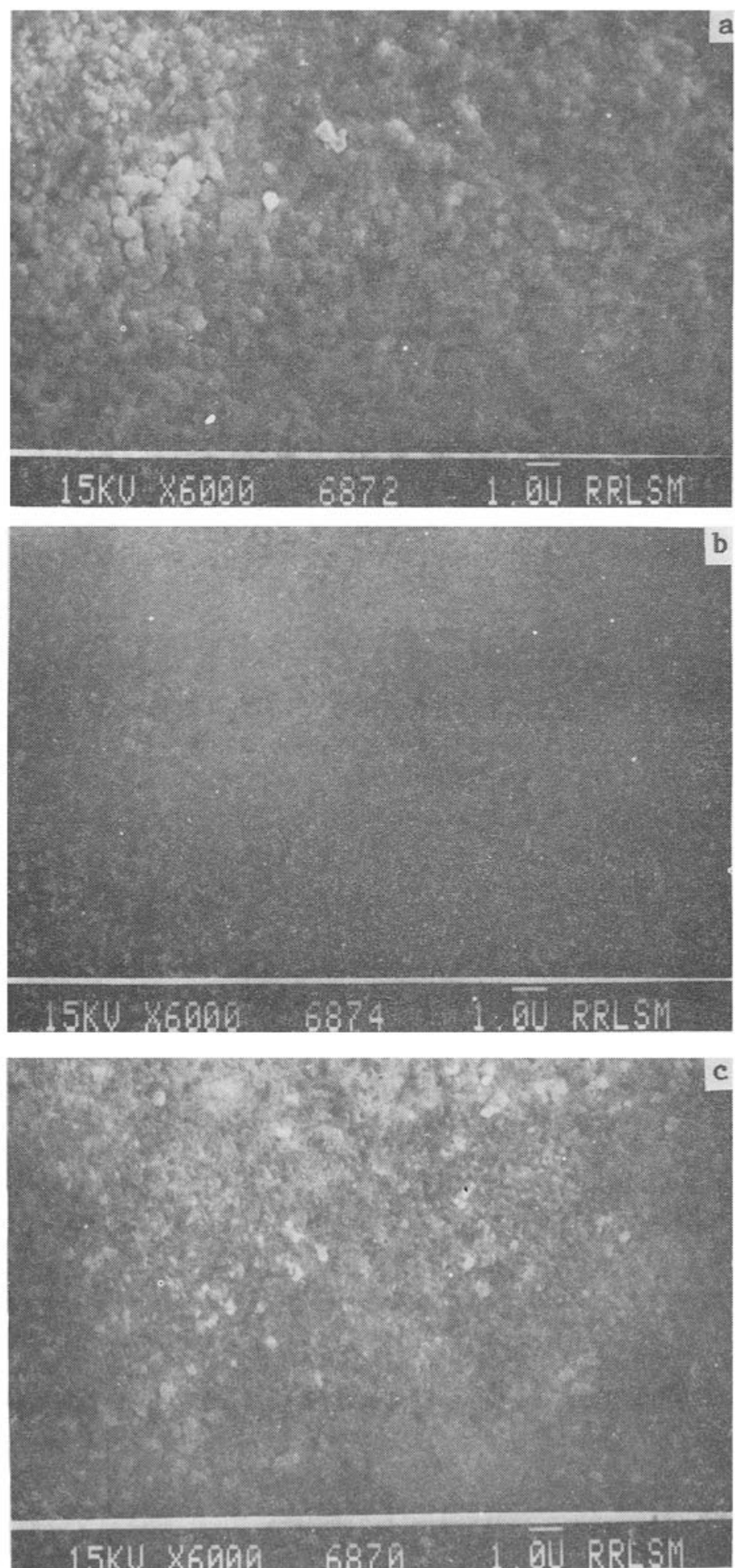

Figure 4. SEM photographs of CdS film prepared at different substrate temperature: (a) film prepared at $200^{\circ} \mathrm{C}$, (b) at $300^{\circ} \mathrm{C}$ and (c) at $360^{\circ} \mathrm{C}$. 
higher rate as the substrate temperature goes above $300^{\circ} \mathrm{C}$. At low temperature large droplets may not get sufficient heat energy to vaporize before reaching the substrate and hence comparatively large droplets reach the substrate. This results in a higher deposition rate and also makes the film surface rather rough. This is shown by the VASE measurements and the roughness has a high value of $\sim 65 \mathrm{~nm}$ at $200^{\circ} \mathrm{C}$. It is also clear from the SEM photograph (figure 4a).

At moderately high temperature (i.e. in the range $280-300^{\circ} \mathrm{C}$ ) the droplets reaching the substrate may get reduced due to the vaporization and this may result in the reaction (leading to the formation of $\mathrm{CdS}$ ) taking place completely over the substrate. This may be the reason for smoothness of the surface of film prepared in this temperature range, as revealed by the ellipsometric study as well as SEM (figures 2 and $3 \mathrm{~b}$ ). But at very high temperature considerable amount of the solvent is vaporized far away from the substrate. The chemical reaction leading to the formation of $\mathrm{CdS}$ will then occur in the vapour phase and this does not contribute to the growth of the film over the substrate surface resulting in a drastic reduction in film deposition rate. Moreover the fine particles of $\mathrm{CdS}$ thus formed may be adhering to the film surface making it rough again. This is also clear from the VASE studies as shown in figure 2. The SEM photograph shown in figure $4 c$ also confirms the VASE measurements on surface roughness.

\section{Conclusion}

CdS films were prepared by spray pyrolysis technique over glass substrate at different substrate temperatures ranging from 200 to $360^{\circ} \mathrm{C}$. VASE was used for the analysis of CdS thin films. Ellipsometric studies show that the films prepared at low and very high temperatures have rough surface layer, but films prepared at $280-300^{\circ} \mathrm{C}$ have very smooth texture with top rough layer having a thickness of $\sim 27 \mathrm{~nm}$. The growth rate is also affected by the substrate temperature. When the substrate temperature is increased from $200^{\circ} \mathrm{C}$ to $300^{\circ} \mathrm{C}$ the growth rate decreases rather slowly. But as the temperature goes above $300^{\circ} \mathrm{C}$ the decrease becomes faster. From this study it becomes clear that the films prepared at about $280-300^{\circ} \mathrm{C}$ have good optical and physical qualities than others and hence these films are best suited for solar cell applications.

\section{Acknowledgement}

One of the authors (SM) is thankful to CSIR, New Delhi, for financial support. Financial assistance from DAE (Government of India) is also gratefully acknowledged. We are thankful to Dr P S Mukherjee, Regional Research Lab, Trivandrum, for SEM analysis.

\section{References}

Albin D S and Risbud S H 1987 Thin Solid Films 147203

Arita T, Hanafusa H, Kitamura S and Takakura H 1991 Conf. Records of 22nd Photovoltaic specialist conference. Las Vegas, NV, USA, p. 946

Azzam R M A and Bashara N M 1977 Ellipsometry and polarized light (Amsterdam: North Holland) Chapter 4 
Banerjee A, Nath P, Vankar V D and Chopra K L 1978 Phys. Status Solidi (a) 46723

Basol B M, Kapur V K and Halani A 1991 Conf. Records of 22nd Photovoltaic specialist conference. Las Vegas, NV, USA p. 893

Bruggeman D A G 1935 Ann. Phys. Leipzig 24636

Cameron D C, Duncan W and Tsang W M 1979 Thin Solid Films 5861

Chopra K L and Das S R 1983 Thin film solar cells (New York: Plenum Press) p. 297

Chow L W, Lee Y C and Lee Kwok H L 1981 Thin Solid Films 81307

Escosura L, Garcia-Camarero E, Arjona F and Rueda F 1984 Solar Cells 11211

Gupta B K and Agnihotri O P 1978 Philos. Mag. B37 631

Kakayama N, Matasumoto H, Yamaguchi K and Iegami I S 1976 Jpn. J. Appl. Phys. 152281

Kaur I, Pandya D K and Chopra K L 1981 J. Electrochem. Soc. 127943

Kwok H L 1980 J. Phys. D. 131911

Nolly J, Abdullah K K and Vijayakumar K P 1987 Phys. Status Solidi (a) 101 K35

Shay J L, Wagneer S, Bachman K, Buchler E and Kaspor H M 1975 Proc. 11th Photovoltaic specialist conference, Phoenix, New York, p. 503

Sunny M and Vijayakumar K P 1994 Bull. Mater. Sci. 17235

Valyommana A G, Vijayakumar K P and Purushothaman C 1992 J. Mater. Sci. Lett. 11616

Vedam K, Mc Marr P J and Narayan J 1985 Appl. Phys. Lett. 47339

Vijayakumar K P 1991a J. Appl. Phys. 696771

Vijayakumar K P 1991 b Bull. Mater. Sci. 1457 